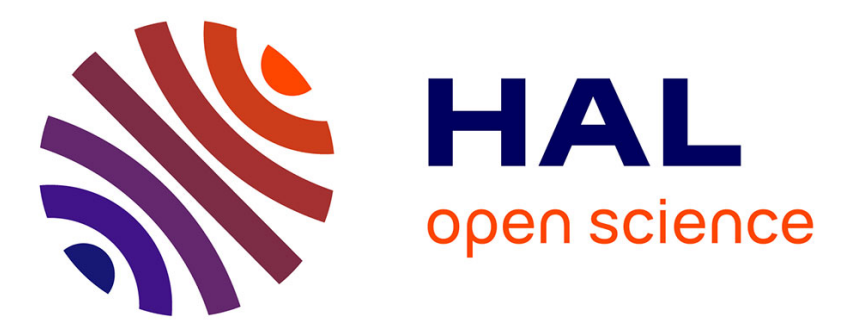

\title{
Muon Production Height Studies with the Air Shower Experiment KASCADE-Grande
}

W.D. Apel, J.C. Arteaga, K. Bekk, M. Bertaina, J. Blümer, H. Bozdog, I.M. Brancus, P. Buchholz, C. Büttner, E. Cantoni, et al.

\section{- To cite this version:}

W.D. Apel, J.C. Arteaga, K. Bekk, M. Bertaina, J. Blümer, et al.. Muon Production Height Studies with the Air Shower Experiment KASCADE-Grande. Astroparticle Physics, 2010, 34 (6), pp.476. 10.1016/j.astropartphys.2010.10.016 . hal-00710473

\section{HAL Id: hal-00710473 https://hal.science/hal-00710473}

Submitted on 21 Jun 2012

HAL is a multi-disciplinary open access archive for the deposit and dissemination of scientific research documents, whether they are published or not. The documents may come from teaching and research institutions in France or abroad, or from public or private research centers.
L'archive ouverte pluridisciplinaire HAL, est destinée au dépôt et à la diffusion de documents scientifiques de niveau recherche, publiés ou non, émanant des établissements d'enseignement et de recherche français ou étrangers, des laboratoires publics ou privés. 


\section{Accepted Manuscript}

Muon Production Height Studies with the Air Shower Experiment KASCADEGrande

W.D. Apel, J.C. Arteaga, K. Bekk, M. Bertaina, J. Blümer, H. Bozdog, I.M. Brancus, P. Buchholz, C. Büttner, E. Cantoni, A. Chiavassa, F. Cossavella, K. Daumiller, V. de Souza, F. Di Pierro, P. Doll, R. Engel, J. Engler, M. Finger, D. Fuhrmann, P.L. Ghia, H.J. Gils, R. Glasstetter, C. Grupen, A. Haungs, D. Heck, J.R. Hörandel, T. Huege, P.G. Isar, K.-H. Kampert, D. Kang, D. Kickelbick, H.O. Klages, K. Link, M. Ludwig, P. Łuczak, H.J. Mathes, H.J. Mayer, M. Melissas, J. Milke, B. Mitrica, C. Morello, G. Navarra, S. Nehls, R. Obenland,

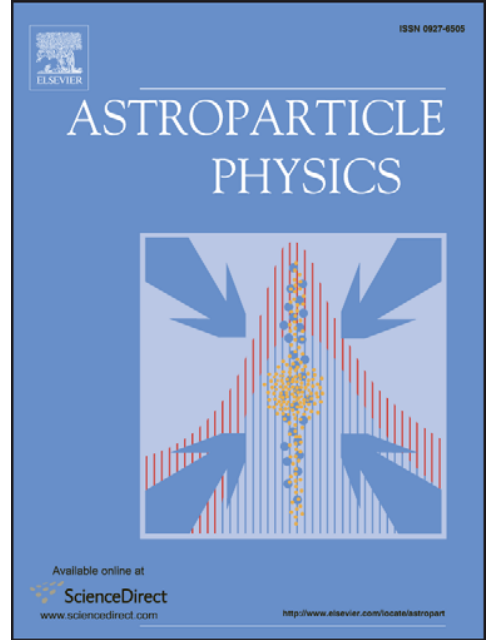
J. Oehlschläger, S. Ostapchenko, S. Over, N. Palmieri, M. Petcu, T. Pierog, H. Rebel, M. Roth, G. Schatz, H. Schieler, F. Schröder, O. Sima, G. Toma, G.C. Trinchero, H. Ulrich, A. Weindl, J. Wochele, M. Wommer, J. Zabierowski

PII: S0927-6505(10)00213-6

DOI: 10.1016/j.astropartphys.2010.10.016

Reference: ASTPHY 1541

To appear in: Astroparticle Physics

Received Date: 7 May 2010

Revised Date: 25 October 2010

Accepted Date: 25 October 2010

Please cite this article as: W.D. Apel, J.C. Arteaga, K. Bekk, M. Bertaina, J. Blümer, H. Bozdog, I.M. Brancus, P. Buchholz, C. Büttner, E. Cantoni, A. Chiavassa, F. Cossavella, K. Daumiller, V. de Souza, F. Di Pierro, P. Doll, R. Engel, J. Engler, M. Finger, D. Fuhrmann, P.L. Ghia, H.J. Gils, R. Glasstetter, C. Grupen, A. Haungs, D. Heck, J.R. Hörandel, T. Huege, P.G. Isar, K.-H. Kampert, D. Kang, D. Kickelbick, H.O. Klages, K. Link, M. Ludwig, P. Łuczak, H.J. Mathes, H.J. Mayer, M. Melissas, J. Milke, B. Mitrica, C. Morello, G. Navarra, S. Nehls, R. Obenland, J. Oehlschläger, S. Ostapchenko, S. Over, N. Palmieri, M. Petcu, T. Pierog, H. Rebel, M. Roth, G. Schatz, H. Schieler, F. Schröder, O. Sima, G. Toma, G.C. Trinchero, H. Ulrich, A. Weindl, J. Wochele, M. Wommer, J. Zabierowski, Muon Production Height Studies with the Air Shower Experiment KASCADE-Grande, Astroparticle Physics (2010), doi: 10.1016/j.astropartphys.2010.10.016

This is a PDF file of an unedited manuscript that has been accepted for publication. As a service to our customers we are providing this early version of the manuscript. The manuscript will undergo copyediting, typesetting, and 
review of the resulting proof before it is published in its final form. Please note that during the production process errors may be discovered which could affect the content, and all legal disclaimers that apply to the journal pertain. 


\section{Muon Production Height Studies with the Air Shower Experiment KASCADE-Grande}

W.D. Apel ${ }^{\mathrm{a}}$, J.C. Arteaga ${ }^{\mathrm{b}, 1}$, K. Bekk ${ }^{\mathrm{a}}$, M. Bertaina ${ }^{\mathrm{c}}$, J. Blümer ${ }^{\mathrm{a}, \mathrm{b}}$, H. Bozdog ${ }^{\mathrm{a}}$, I.M. Brancus ${ }^{\mathrm{d}}$, P. Buchholz ${ }^{\mathrm{e}}$, C. Büttner ${ }^{\mathrm{b}}$, E. Cantoni ${ }^{\mathrm{c}, \mathrm{f}}$, A. Chiavassa ${ }^{c}$, F. Cossavella ${ }^{\mathrm{b}, 2}$, K. Daumiller ${ }^{\mathrm{a}}$, V. de Souza ${ }^{\mathrm{b}, 3}$,

F. Di Pierro ${ }^{c}$, P. Doll*,a, R. Engel ${ }^{\mathrm{a}}$, J. Engler ${ }^{\mathrm{a}}$, M. Finger ${ }^{\mathrm{a}}$, D. Fuhrmann ${ }^{\text {, }}$, P.L. Ghia ${ }^{\mathrm{f}}$, H.J. Gils ${ }^{\mathrm{a}}$, R. Glasstetter ${ }^{\mathrm{g}}$, C. Grupen ${ }^{\mathrm{e}}$, A. Haungs ${ }^{\mathrm{a}}$, D. Heck ${ }^{\mathrm{a}}$, J.R. Hörandel ${ }^{\mathrm{b}, 4}$, T. Huege ${ }^{\mathrm{a}}$, P.G. Isar ${ }^{\mathrm{a}, 5}$, K.-H. Kampert', D. Kang ${ }^{\mathrm{b}}$, D. Kickelbick ${ }^{\mathrm{e}}$, H.O. Klages ${ }^{\mathrm{a}}$, K. Link ${ }^{\mathrm{b}}$, M. Ludwig, ${ }^{\mathrm{b}}$, P. Luczak ${ }^{\mathrm{h}}$, H.J. Mathes ${ }^{\mathrm{a}}$, H.J. Mayer ${ }^{\mathrm{a}}$, M. Melissas ${ }^{\mathrm{b}}$, J. Milke, B. Mitrica ${ }^{\mathrm{d}}$,

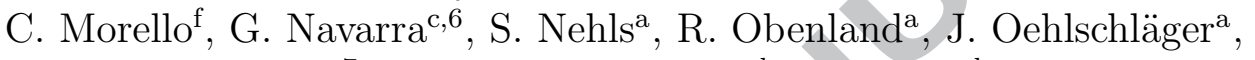

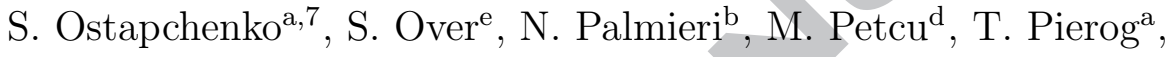
H. Rebel ${ }^{\mathrm{a}}$, M. Roth ${ }^{\mathrm{a}}$, G. Schatz ${ }^{\mathrm{a}}$, H. Schieler ${ }^{\mathrm{a}}$, F. Schröder ${ }^{\mathrm{a}}$, O. Sima ${ }^{\mathrm{i}}$, G. Toma ${ }^{\mathrm{d}}$, G.C. Trinchero ${ }^{\mathrm{f}}$, H. Ulrich ${ }^{\mathrm{a}}$, A. Weindl ${ }^{\mathrm{a}}$, J. Wochele ${ }^{\mathrm{a}}$, M. Wommer ${ }^{\mathrm{a}}$, J. Zabierowski*, h

${ }^{a}$ Institut für Kernphysik, Karlsruher Institut für Technologie - Campus Nord, 76021 Karlsruhe, Germany

${ }^{b}$ Institut für Experimentelle Kernphysik, Karlsruher Institut für Technologie - Campus Süd, 76021 Karlsruhe, Germany

${ }^{c}$ Dipartimento di Física Generale dell'Università, 10125 Torino, Italy

${ }^{d}$ National Institute of Physics and Nuclear Engineering, 7690 Bucharest, Romania

${ }^{e}$ Fachbereich Physik, Universität Siegen, 57068 Siegen, Germany

${ }^{f}$ Istituto di Fisica dello Spazio Interplanetario, INAF, 10133 Torino, Italy

${ }^{g}$ Fachbereich Physik, Universität Wuppertal, 42097 Wuppertal, Germany

${ }^{h}$ The Andrzej Soltan Institute for Nuclear Studies, 90950 Lodz, Poland

${ }^{*}$ Corresponding authors. Address: Institut für Kernphysik, Karlsruher Institut für Technologie (KIT), Post Box 3640, 76021 Karlsruhe, Germany, Tel.: +49 7247 824171; fax:+49 7247823321

Email addresses: paul.doll@kit.edu (P. Doll), janzab@zpk.u.lodz.pl

(J. Zabierowski)

${ }^{1}$ now at: Instituto de Física y Matematicas, Universidad Michoacana, Morelia, Mexico

${ }^{2}$ now at: Max-Planck-Institut für Physik, München, Germany

${ }^{3}$ now at: Universidade de São Paulo, Instituto de Física de São Carlos, Brasil

${ }^{4}$ now at: Dept. of Astrophys. Radboud University Nijmegen, The Netherlands

${ }^{5}$ now at: Institute for Space Sciences, Bucharest-Magurele, Romania

${ }^{6}$ Deceased

${ }^{7}$ now at: University of Trondheim, Norway 
${ }^{i}$ Department of Physics, University of Bucharest, 76900 Bucharest, Romania

\section{Abstract}

A large area $\left(128 \mathrm{~m}^{2}\right)$ muon tracking detector, located within the KASCADE experiment, has been built with the aim to identify muons $\left(E_{\mu} \geqslant 0.8 \mathrm{GeV}\right)$ and their angular correlation in extensive air showers by track measurements under 18 r.l. shielding. Orientation of the muon track with respect to the shower axis is expressed in terms of the radial and tangential angles, which are the basic tools for all muon investigations with the tracking detector. By means of triangulation the muon production height is determined. Distributions of measured production heights are compared to CORSIKA shower simulations. Analysis of these heights reveals a transition from light to heavy cosmic ray primary particles with increasing shower energy in the energy region of the 'Knee' of the cosmic ray spectrum.

Key words: Cosmic rays, Air shower, Muons, Muon production height

\section{Introduction}

Ultra High Energy (UHE) astrophysics includes, as one of its topics, the 3 investigation of UHE Cosmic Ray (CR) particles interacting with the nu4 clei of the atmosphere and creating Extensive Air Showers (EAS) which are 5 studied by means of ground based experiments of large detection area. To understand the nature of UHE particles it is necessary to measure as many components of the EAS cascade as possible. Particularly important is the measurement of the muon component, because muons, being a result of decays of mesons - the most numerous products of hadronic interactions in an air shower - carry information about those interactions to the observation level. In turn, knowledge of UHE hadronic interactions is a necessary condition for answering many questions formulated in CR research. The angular correlation information on EAS muons measured with the tracking detector is especially closely correlated to the parameters of the interactions in question. A recent compilation of muon study results is given in Ref. [1].

In the past, various tracking detectors for measuring CR particles have been put into operation either on the surface of Earth [2]-[8] or deep under- 
ground [9]. Currently active installations for muon and neutrino studies are IceCube [10], ANTARES [11] and MINOS [12]. Tracking detectors provide the possibility to study the muon production height which is the subject of this work.

Muon information has usually been integrated over a large sample of showers and over the whole longitudinal profile. However, muons have some advantage compared with optical photons [13]-[15]: they reflect the development of the nuclear cascade with no mediation from electromagnetic cascades and, similar to the radio emission [16], they can be measured 24 hours a day not only on clear moonless nights. Their evident disadvantage is that muons are less numerous than photons and are, therefore, subject to large fluctuations. In addition, they are charged particles and are subject to deflection in the geomagnetic field and also suffer from multiple Coulomb scattering in the atmosphere and detector shielding.

Muon tracking allows to measure the composition sensitive profile of a shower in the 'Knee' region, where it is not possible by the fluorescence technique used, e.g. by Fly's Eye [17] and Auger [18] experiments. It enables as well the study of hadron interactions $[19,20]$. Muons have never been used up to now, with sufficient accuracy and large statistics, for the reconstruction of the longitudinal development of the EAS hadron component of individual showers due to the difficulty of building large area, ground-based muon telescopes. The reconstruction of the longitudinal development of the muon component by means of triangulation [21]-[24] provides a powerful tool for primary mass estimation and for study of high-energy hadron interactions with atmospheric nuclei.

The KASCADE-Grande air-shower experiment [25], set-up in Karlsruhe, Germany provides information on individual EAS up to primary energy $10^{18} \mathrm{eV}$. Complex analyses of KASCADE data [26] resulted in CR flux spectra for five groups of primary masses over the 'Knee' region. However, these spectra exhibit a strong dependence on the hadronic interaction models used in the data analysis. Therefore, an independent investigation of additional shower observables like muon angular correlations with respect to the shower direction is of a great importance. To address this task a Muon Tracking Detector (MTD) [27] was constructed providing additional information on muons for the measured EAS.

In this work we present the experimental investigation of the hadronic cascade in EAS using tracks of muons measured at the KASCADE-Grande experiment. Precise angular information on those tracks is used to determine 
muon production heights. The sensitivity of this quantity to the mass and energy of CR primary particles is shown. The validity of hadronic interaction models used in Monte Carlo simulations is discussed. Due to the energy dependence of muon production height a transformation to muon production depth is used to investigate, in a model independent way, the CR composition. An estimate of the composition covering two decades of primary energy around the 'Knee' is given.

\section{Muon Tracking Detector (MTD) in KASCADE-Grande}

The original KASCADE experiment [28], with its array of scintillation counters registering the electron and muon components in EAS has been enlarged into KASCADE-Grande in 2001 - 2003 by adding the extended 'Grande' array of scintillation counters. The MTD was put into operation and started regular data taking in the beginning of 2003. It provides additional information on muons for the two parts of the experiment: KASCADE and Grande. Both arrays trigger the MTD.

The MTD utilizes streamer tube (ST) gas detectors grouped in modules. Four modules, three positioned on horizontal planes (top, middle, bottom) and one arranged vertically (wall), form a muon telescope, called a detector tower. The whole MTD comprises 16 towers arranged in two rows of a total detection area of $128 \mathrm{~m}^{2}$. A multi-layer filter of six $3 \mathrm{~cm}$ thick iron plates, separated each by $5 \mathrm{~cm}$ of sand, covered with soil and resting on $30 \mathrm{~cm}$ of steel-concrete roof, absorbs a large fraction of accompanying lowenergy particles, thus enhancing the identification of muons with an energy exceeding $0.8 \mathrm{GeV}$. The details of the design and performance of the MTD can be found elsewhere [27, 29].

The MTD shows a good stability of operation. Hereby, the stability of the gas composition on the sub-percent level is of great importance. However, the open gas system is influenced by the atmospheric pressure and temperature (internal in the detector $T_{\text {int }}$ and atmospheric $T_{a t m}$ ), which is observed in the measured free monitor muon count rate. After applying corrections, the free monitor rate $(R)$ has a remaining variation of about $1 \%$. The resulting temperature and pressure coefficients of the rate, together with the gas composition stability parameters, are given in Table 1 . They provide the parameters for the MTD track efficiency determination and correction for temperature and pressure, i.e. for varying atmospheric conditions at KASCADE-Grande. 


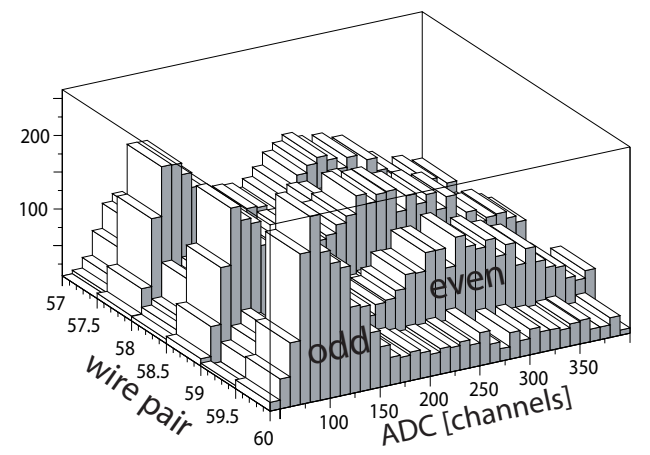

Figure 1: Anode wire amplitude spectra for individual odd/even numbered wire cells.

The analysis is based on 3-hit tracks, which are derived from $x$ and $y$ (wire and strip) hits in the 3 horizontal modules for each tower. Using 3-hit tracks only, together with the track quality parameter discussed in Ref. [27], makes the amount of tracks uncorrelated with a shower being below $1 \%$. The modules show, after correction for geometry, pressure and temperature variations, a hit efficiency close to $100 \%$, varying on average by less than $1 \%$. The track efficiency $(\epsilon)$ stability parameter is given in Table 1 . The cluster sizes are limited to $10 \mathrm{~cm}$ at the wires and to $16 \mathrm{~cm}$ at the strips to control punch-through effects and to achieve tracks of a good quality. More details on track quality and its influence on the direction determination and comparison with shower and detector simulations can be found in Refs. [27], [30] and [31].

Previously, the anode wire readout in the MTD was operated by combining two neighboring wire cells. To improve the angular resolution a modification was introduced [32], enabling to identify even and odd numbered wires, using the same common readout electronic channel. The individual wire cells are identified by means of the pulse shape, as illustrated in Fig. 1. This procedure improves the angular resolution by $30 \%$.

\section{Tangential and Radial Angles}

The KASCADE-Grande data, both from the KASCADE and 'Grande' detector arrays, allow us, after detailed analysis steps [33], [34], [35] to determine the direction $(\theta$ and $\phi)$, the shower core position, the total number of electrons - electron size $\lg \left(N_{e}\right)$, and the total number of muons - muon size $\lg \left(N_{\mu}\right)$, for each shower. In KASCADE the so-called 'truncated' muon 
Table 1: Variation of track efficiency $(\epsilon)$, Monitor Rate $(R)$ and Gas Composition Stability Parameters of the MTD

\begin{tabular}{lc}
\hline \hline$d \epsilon / d T_{\text {int }}$ & $+(0.16 \pm 0.02) \% /{ }^{\circ} \mathrm{C}$ \\
\hline$d \epsilon / d p_{\text {int }}$ & $-(0.12 \pm 0.04) \% / \mathrm{mb}$ \\
\hline$d R / d T_{a t m}$ & $-(0.31 \pm 0.02) \% /{ }^{\circ} \mathrm{C}$ \\
\hline$d R / d p_{a t m}$ & $-(0.12 \pm 0.04) \% / \mathrm{mb}$ \\
\hline Argon & $13.8 \pm 0.1 \%$ \\
$\mathrm{CO}_{2}$ & $54.0 \pm 0.1 \%$ \\
Isobutane & $30.2 \pm 0.1 \%$ \\
Ethanol & $2.0 \pm 0.2 \%$ \\
\hline \hline
\end{tabular}

number $\lg \left(N_{\mu}^{t r}\right)$, is evaluated in the distance range $40-200 \mathrm{~m}$ from the shower core. In 'Grande' the muon size is determined in a different way [35]. The 'truncated' muon number is approximately related to the total muon number in the following way: $\lg \left(N_{\mu}\right) \approx \lg \left(N_{\mu}^{t r}\right)+0.5$ [36]. This relation, obtained by simulations, results for the same showers reconstructed with KASCADE and Grande separately in similar muon shower size $\lg \left(N_{\mu}\right)$ values within 10-15\%.

Determination of primary cosmic ray energy from the parameters of registered air showers is of primary interest for all EAS experiments. In KASCADE-Grande total energy $E_{0}$ of the primary CR particle, in $\mathrm{GeV}$, can be expressed in an almost mass independent manner through $\lg \left(N_{e}\right)$ and $\lg \left(N_{\mu}\right)$. Based on extensive simulation studies [37] with CORSIKA [38], using QGSJet01 [39] the following formula is found

$$
\lg \left(E_{0}\right)=a \cdot \lg \left(N_{e}\right)+b \cdot \lg \left(N_{\mu}\right)+c,
$$

where the parameters have the following values. For the KASCADE array data analysis $a=0.19, b=0.79$ and $c=2.33$, and in addition, $\lg \left(N_{\mu}^{t r}\right)$ is used instead of $\lg \left(N_{\mu}\right)$. For the Grande array analysis, following Ref. [40], we have $a=0.31, b=0.67$ and $c=1.85$. Note, that because of the large coefficient for $\lg \left(N_{\mu}\right)$ this parameter is sometimes used to demonstrate the behavior of various shower quantities as a function of primary energy, where an increase in $\lg \left(N_{\mu}\right)$ means increase in $E_{0}$. Formula $(1)$ gives $\approx 10-20 \%$ (depending on the primary energy range) uncertainty in energy determination which is sufficient for this study. Investigation of an improved determination of $E_{0}$ in KASCADE-Grande is in progress and first results can be found in [41] and 
137

references therein.

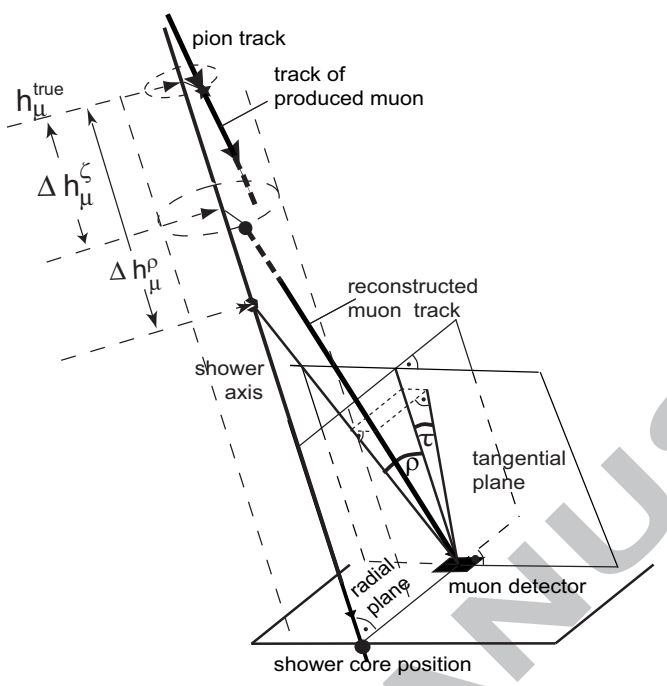

Figure 2: Illustration of the angular correlations in EAS and the definition of the radial $(\rho)$ and tangential $(\tau)$ angles. The differences between the true and the reconstructed $h_{\mu}$ values for the two reconstruction approaches - see text - are also shown.

For the determination of the muon production height $h_{\mu}$ in EAS, orientation of the muon track with respect to the shower axis has to be investigated. Due to their transverse momentum parent mesons travel away from the shower axis. To investigate angular correlation of the muon track with respect to the shower axis, the angle in space between both directions is decomposed into two components: the radial $(\rho)$ and the tangential $(\tau)$ angles [27], [42], shown in Fig. 2. The $\rho$ angle is defined in the radial plane, subtended by the shower axis, the shower core position and the point where the muon hits the MTD detector plane. The $\tau$ angle is defined in the tangential plane which is perpendicular to the radial plane and parallel to the shower axis, and goes also through the point where the muon hits the detector plane. The value of $\tau$ reflects predominantly the amount of muon scattering in the atmosphere and in any relevant absorber/detector material, and to some extent also the fact that muons can be produced off the shower axis. A contribution from the deflection of high-energy muons in the geomagnetic field (locally $<0.1^{\circ}[31]$ ) is also included there. The $\tau$ angle distribution, when averaged over all azimuth angles, is symmetrical around zero and gets 


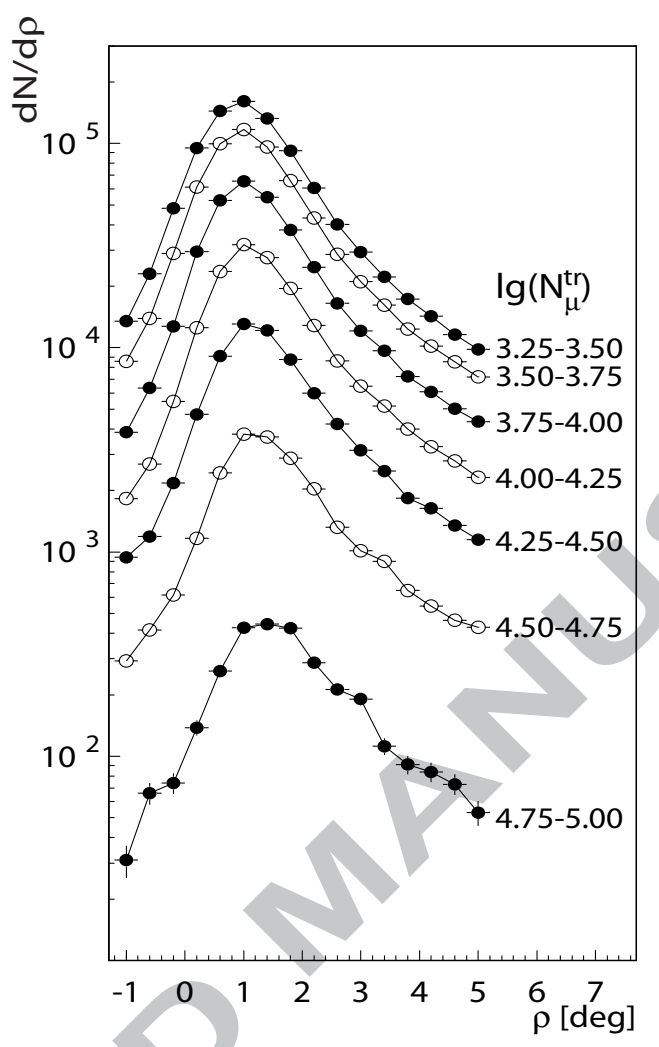

Figure 3: An example of radial angle distributions from Ref. [31] shows the behavior of this quantity in a wide range of $\lg \left(N_{\mu}^{t r}\right)$, i.e. of primary energy. The selection used comprises a radial distance range $40-120 \mathrm{~m}$, shower zenith angles $\theta<30^{\circ}$ and $|\tau|<0.7^{\circ}$. Relative yields reflect the $\mathrm{CR}$ flux in different $\lg \left(N_{\mu}^{t r}\right)$ intervals. Lines connect the data points to guide the eyes.

narrower with increasing muon momentum [43], which is expected from the momentum dependence of multiple scattering and from geomagnetic bending. The $\rho$ angle value, being defined in the shower coordinate system, is dominantly correlated with the transverse momentum of the parent meson. It is also substantially larger than the corresponding $\tau$ values. The multiple scattering of the muon in the atmosphere and muon filter above the MTD contributes also to the value of $\rho$, but has a relatively minor effect on it. An example of $\rho$ distributions are shown in Fig. 3 [31]. They exhibit an asymmetric shape, being shifted with increasing $\lg \left(N_{\mu}^{t r}\right)$ towards larger $\rho$ values. This shift is due to the deeper penetration of higher energy showers, 
but it is not so strongly pronounced because of the wide range of shower core distances selected for Fig. 3. Negative $\rho$ values are due to a finite resolution of the $\rho$ angle $\left(\approx 0.2^{\circ}\right)$ and due to the possible muon scattering towards the shower axis.

\section{Muon Production Height}

In previous theoretical studies [44] the longitudinal development of the muon component in EAS was investigated by an integral transformation from lateral particle densities to a more shower development related observable like the muon production heights. Due to the very different shower development for light and heavy primary CR particles, their corresponding mean production heights are expected to be different, and thus to be indicative for the primary mass (see Fig. 4).

Conditions of the experiment and the response of the MTD were extensively studied with experimental data and simulations ([27], [30], [31]) to ensure the best tracking resolution and to clean the sample from nonshower-muon tracks. The effects of the non-central location of the MTD within KASCADE were also taken into account.

In order to decrease the effect of multiple muon scattering on their path to the detector $|\tau|$ angles smaller than $0.7^{\circ}$ are considered. This cut enriches the sample with high energy muons, above a few $\mathrm{GeV}$ [30], having smaller multiple scattering angles $\left(\sigma_{\tau} \approx 0.2^{\circ}\right)$ and smaller bending in the geomagnetic field, which improves considerably the determination of the correct production height.

Elimination of tracks not belonging to shower muons or originating in the absorber above the MTD (in addition to the cuts ensuring good track quality, mentioned in Section 2) is achieved by limiting the radial angle value to $\rho<8^{\circ}$. For muon production height analysis in this work only positive radial angles are considered.

To ensure a nearly full azimuthal symmetry of the measured muons, events with $40 \mathrm{~m}-80 \mathrm{~m}$ core distance to the MTD are selected (the location of the MTD is shifted by $54.65 \mathrm{~m}$ to the north from the KASCADE center because this detector was not considered from the beginning). In addition, this selection lowers the influence of the geomagnetic field on the mean muon directions. Below $40 \mathrm{~m}$ the punch-through effects forbid a valuable muon tracking and above $80 \mathrm{~m}$ the highly asymmetric azimuthal event 
distribution (see Fig. 6.3 in Ref. [31]) is difficult to correct. For Grande reconstructed high-energy events the distance range $250 \mathrm{~m}-360 \mathrm{~m}$ was chosen to avoid extended punch-through and trigger efficiency problems for large distances. For KASCADE (Grande) selections a combined Array-MTD analysis is only reliable above shower size $\lg \left(N_{e}\right) \geq 4.8(6.0)$ and $\lg \left(N_{\mu}\right) \geq 4.1$ (5.5).

In this work the experimental data on showers with zenith angles $\theta<18^{\circ}$ were analyzed. The mean height values $\left\langle h_{\mu}\right\rangle$ were calculated in the altitude range up to $12 \mathrm{~km}$ along the shower axis, where the majority of muons with energy exceeding $0.8 \mathrm{GeV}$ at ground are produced. For example, as CORSIKA simulations show, in a $10^{16} \mathrm{eV}$ proton induced shower about $90 \%$ of such muons are produced in this altitude range. Despite of the fact that the first encounters with nuclei of the atmosphere occur at even higher altitudes, they are not considered in this work, both in data and simulations, because of their large uncertainty. The value of $12 \mathrm{~km}$ was chosen because at this altitude, and for the selected distance range of investigated muons to the shower core, the uncertainties in the $\rho$ angle values become comparable to the values themselves.

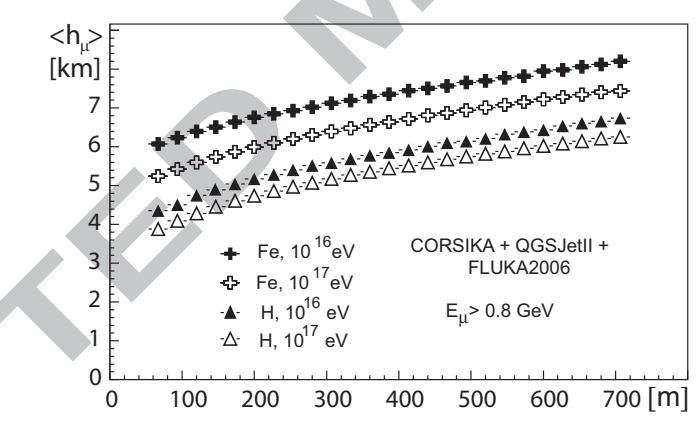

Figure 4: Mean muon production heights observed at different distances from the shower core in CORSIKA simulated vertical showers for proton and iron primaries and two fixed primary energy values [47].

Employing the $\rho$ and $\tau$ angles to define the orientation of muon track with respect to the shower axis together with the relative distance $R_{\mu}$ from the muon hit to the shower core position, the height $h_{\mu}$ can be calculated. This can be done along the muon track, which is the appropriate way to consider absorption or interaction of muons in showers [45, 46].

Another approach, used in the present work, is the determination of the height $h_{\mu}$ along the shower axis. One way is to use the relation $h_{\mu}=R_{\mu} / \tan \rho$ 
in the shower coordinate system. We could also employ, for angles much smaller than one radian, instead of $\rho$, the angle $\zeta=\sqrt{\left(\rho^{2}+\tau^{2}\right)}$ [43], which is the angle in space between muon track and the shower direction.

As shown in Fig. 2 both reconstruction methods lead to muon production heights different from the true one by $\Delta h_{\mu}^{\rho}$ in case of the calculation with $\rho$, and by $\Delta h_{\mu}^{\zeta}$ when using the angle $\zeta$. In the former case the muons are assumed to be produced on the shower axis (which is not always the case) while in the latter, the muon production point is reconstructed on a cylindrical surface around the shower axis. The actual value of this difference in both reconstruction methods (being one of the sources of systematic errors) varies slightly with the type of primary and its energy, leading in most cases to an underestimation of the mean production height by a few percent.

In Table 2 a comparison of these differences is given for two fixed primary energies and two primary species. The results for muons with $E_{\mu}>0.8 \mathrm{GeV}$ are obtained with CORSIKA simulated vertical showers (500 showers for $10^{15} \mathrm{eV}$ and 158 for $10^{16} \mathrm{eV}$ primary energy) using the QGSJet01 [39] and GHEISHA [48] interaction models. As seen in columns 3 and 4 the uncertainties range from $\approx 2 \%$ to $\approx 8 \%$, showing slightly better results for the ' $\rho$-method'.

One can expect smaller errors if a correction for multiple scattering in the atmosphere was made in the calculations. The actual amount of scattering is, of course, not known. However, for the selection used in the analysis $\left(0<\rho<8^{\circ}\right.$ and $\left.|\tau|<0.7^{\circ}\right)$ the simulations predict an average scattering angle at the level of $0.2^{\circ}$. It was checked with Monte Carlo simulations that up to $12 \mathrm{~km}$ altitude and a distance range of 40-80 m between a muon and the shower core, a subtraction of the absolute value of $\tau$ from $\rho$ or $\zeta$ when calculating $h_{\mu}$ provides an improvement of the accuracy. Reconstructed negative $h_{\mu}$ values are below $5-7 \%$. The difference between the reconstructed mean value of the positive $h_{\mu}$ and the true mean muon production height is illustrated in columns 5 and 6 of Table 2. The investigation of $h_{\mu}$ in this work was done using the following relation

$$
h_{\mu}=\frac{R_{\mu}}{\tan (\rho-|\tau|)} .
$$

The mean muon production height reconstructed with the relation (2) differs at most by $\pm 4 \%$ from the true value.

In Fig. 5 an example of muon production height distributions $h_{\mu}$ (from Ref. [31]), obtained with KASCADE data, shows the behavior of this quantity 
Table 2: Dependence of the systematic mean muon production height errors (in \% of the true value) on the reconstruction method

\begin{tabular}{cccccc}
\hline \hline 1 & 2 & 3 & 4 & 5 & 6 \\
\hline Primary & $E_{0}[\mathrm{eV}]$ & $\Delta h_{\mu}^{\rho}$ & $\Delta h_{\mu}^{\zeta}$ & $\Delta h_{\mu}^{\rho}(\mathrm{cor})$ & $\Delta h_{\mu}^{\zeta}(\mathrm{cor})$ \\
\hline $\mathrm{H}$ & $10^{15}$ & $-3.1 \%$ & $-4.0 \%$ & $+2.1 \%$ & $+2.4 \%$ \\
\hline $\mathrm{Fe}$ & $10^{15}$ & $-7.3 \%$ & $-8.4 \%$ & $-3.5 \%$ & $-3.1 \%$ \\
\hline $\mathrm{H}$ & $10^{16}$ & $-1.8 \%$ & $-2.7 \%$ & $+4.2 \%$ & $+4.3 \%$ \\
\hline $\mathrm{Fe}$ & $10^{16}$ & $-5.0 \%$ & $-5.7 \%$ & $-0.5 \%$ & $+0.02 \%$ \\
\hline \hline
\end{tabular}

in a wide range of primary energy (expressed by $\lg \left(N_{\mu}^{t r}\right)$ ). Event selection differs from the one used for the analysis in this work in order to show some general features of the distributions. These distributions indicate that the production heights may extend to very large values. For large $\lg \left(N_{\mu}^{t r}\right)$ values (large primary energies) the deeper shower development is mostly demonstrated by relatively fewer muons from large $h_{\mu}$.

Further systematic effects are to be considered. In the region of small $\lg \left(N_{\mu}^{t r}\right)$ in Fig. 5, the measured $h_{\mu}$ values can have a bias. For a successful reconstruction of the shower parameters on the KASCADE array level, the showers must have a certain electron particle number $N_{e}$ at ground. At small energies only showers which fluctuate to lower production heights have enough particles and are fully reconstructed. Those showers are very likely proton induced showers, and hence a bias may be present in the analysis for small $\lg \left(N_{\mu}^{t r}\right)$ due to the smaller trigger efficiency for iron, being of about $80 \%$ at the lowest $\lg \left(N_{\mu}^{t r}\right)$ bin.

With increasing shower zenith angle $\theta,\left\langle h_{\mu}\right\rangle$ is shifting somewhat towards larger values, but the change amounts to less than hundred meters in the whole range of $\theta$. Similar shifts are observed for an analysis using full simulation, only. This effect is small compared to the difference of $\left\langle h_{\mu}\right\rangle$ for proton and iron induced showers, which amounts to about $1000 \mathrm{~m}$ (see Fig. 4), when using the cuts employed in our analysis.

\section{Muon Production Heights in Showers Induced by Light and Heavy Primary Masses}

The electron size $\lg \left(N_{e}\right)$ and muon size $\lg \left(N_{\mu}^{t r}\right)$ provide an opportunity to separate light from heavy primary CR initiated showers. The size parameters 


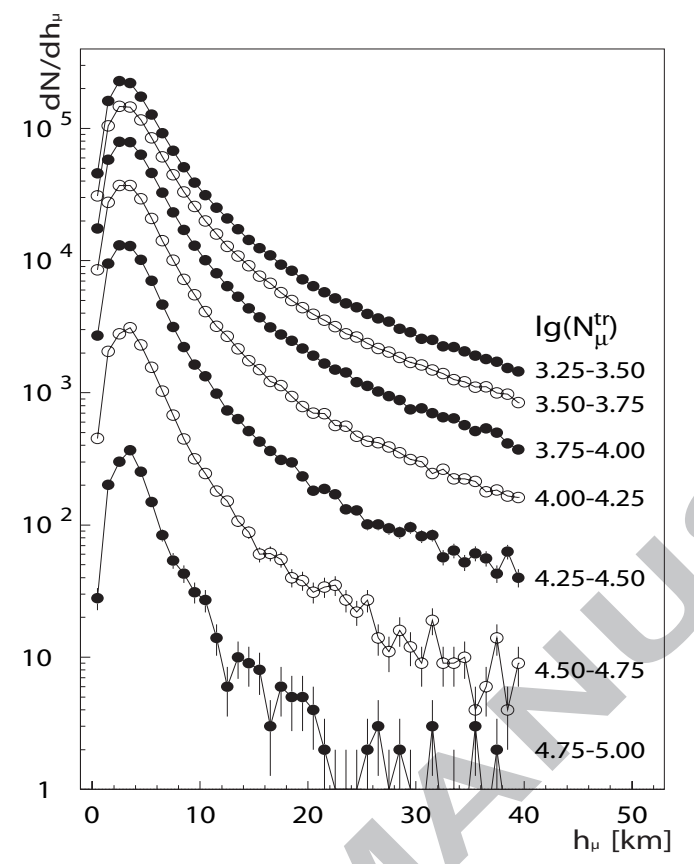

Figure 5: An example of muon production height distributions from Ref. [31] shows the behavior of this quantity in a wide range of $\lg \left(N_{\mu}^{t r}\right)$, i.e. primary energy. The selection used comprises a radial distance range of $40-120 \mathrm{~m}$ and shower zenith angles $\theta<30^{\circ}$. Radial and tangential angle cuts are the same as used in this article $\left(\rho=0-8^{\circ}\right.$ and $\left.|\tau|<0.7^{\circ}\right)$. Relative yields reflect the CR flux in different $\lg \left(N_{\mu}^{t r}\right)$ intervals. Lines connect the data points to guide the eyes.

$\lg \left(N_{e}\right)$ and $\lg \left(N_{\mu}^{t r}\right)$, as determined from the KASCADE array, are for the following studies corrected for their shower angle dependent attenuation, see Ref. [49], employing the formula $\lg \left(N_{i}^{0}\right)=\lg \left(N_{i}(\theta)\right)+0.43 \cdot\left(1022 / \Lambda_{i}\right) \cdot(\sec \theta-$ 1), where $\Lambda_{e}=175 \mathrm{~g} \cdot \mathrm{cm}^{-2}, \Lambda_{\mu}=823 \mathrm{~g} \cdot \mathrm{cm}^{-2}$, and $i$ stands for $e$ or $\mu$, respectively. The ratio of the corrected parameters, namely $\lg \left(N_{\mu}^{t r, 0}\right) / \lg \left(N_{e}^{0}\right)$, turned out to be sensitive to the mass composition of primary CR [33,37].

A ratio $\lg \left(N_{\mu}^{t r, 0}\right) / \lg \left(N_{e}^{0}\right)$ of 0.74 , which corresponds roughly to Nitrogen, is used to divide the experimental shower data into samples enriched with light and heavy primaries $[37,31]$. Grouping the showers in the twoparameter space $\lg \left(N_{e}^{0}\right)$ vs. $\lg \left(N_{\mu}^{t r, 0}\right)$, according to $\lg \left(N_{\mu}^{t r, 0}\right)>0.74 \lg \left(N_{e}^{0}\right)$ or $\lg \left(N_{\mu}^{t r, 0}\right) \leq 0.74 \lg \left(N_{e}^{0}\right)$, heavy or light primary CR particles are selected, respectively. Using this coarse selection of the showers their muon tracks can 
be used to calculate the muon production height $h_{\mu}$ along the shower axis.

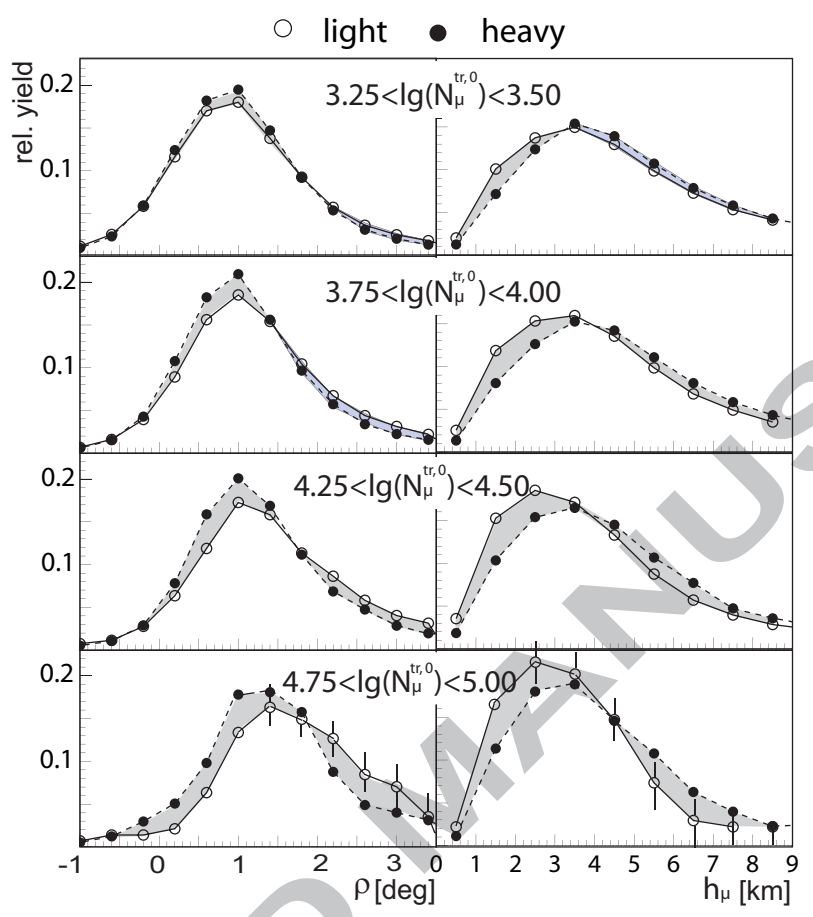

Figure 6: Radial angle distributions (left panel) and muon production height distributions $h_{\mu}$ along the shower axis (right panel) for light and heavy CR primary mass enriched showers and for different $\lg \left(N_{\mu}^{t r, 0}\right)$ intervals. Shower core distances range is $40-80 \mathrm{~m}$, and shower angles $\theta<18^{\circ}$. Lines connect the data points to guide the eyes.

Fig. 6 shows the distributions of the radial angle and muon production height (left and right panel, respectively) for the selected distance range 40$80 \mathrm{~m}$ for different primary energies (expressed in terms of the muon number). Besides the dynamical shift of the radial angle distributions with increasing muon number, the figures show an increasing sensitivity to the mass of the CR particle. The drop to zero yield at small $h_{\mu}$ relates to the cuts on $\rho$ and $\tau$ angles and the $\mu$-hit shower core distance range employed in the analysis.

At high energies large muon production altitudes are predominantly created by heavy primaries. Narrower distributions, differently for light and heavy CR primaries, indicate a decrease of fluctuations in the $\left\langle h_{\mu}>\right.$ parameter with increasing primary energy. Fig. 7 compares the muon production height distributions of Fig. 6 to simulation results for proton (triangles) and 


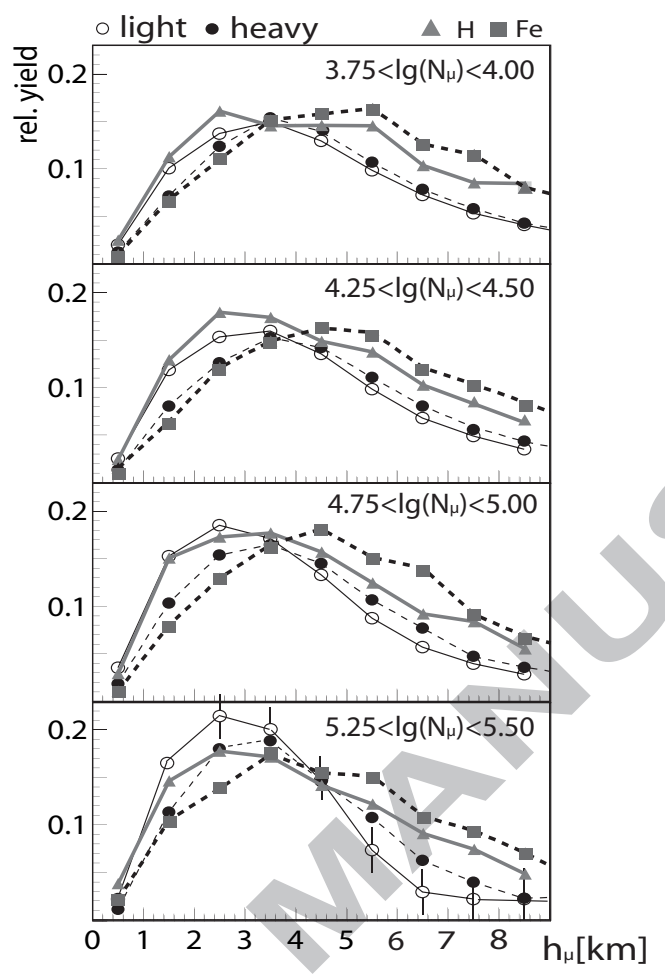

Figure 7: Muon production height distributions along the shower axis $h_{\mu}$ for light (open circles) and heavy (full circles) enriched shower samples and a shower core distance range $40-80 \mathrm{~m}$, and shower zenith angles $\theta<18^{\circ}$ compared to CORSIKA simulations employing QGSjetII + FLUKA models for proton $(\mathrm{H})$ and iron $(\mathrm{Fe})$ primaries. Here $\lg \left(N_{\mu}\right) \approx \lg \left(N_{\mu}^{t r, 0}\right)+0.5$ (see Section 3). Lines connect the data points to guide the eyes.

iron (squares) primaries. The CORSIKA simulations were performed with ver. 6.307 of the code using the QGSJetII and, for interaction energies below $200 \mathrm{GeV}$, FLUKA2002.4 models [50].

Detector simulations were based on GEANT [51]. Identical cuts for data and simulations were used and the slope of the simulated energy spectrum was weighted to -2.7 below and to -3.1 above the 'Knee'. In Fig. 7 (as well as in Fig. 6) the plots of $h_{\mu}$ are normalized to integral yield equal one in the full range up to $12 \mathrm{~km}$, but they are shown to $9 \mathrm{~km}$ only in order to expand the low production height region. However, the following discussion of the features seen at high values of $h_{\mu}$ is also true for the production heights 
above $9 \mathrm{~km}$. In this expanded region we see up to $\approx 3.5 \mathrm{~km}$ that data are embraced by the simulation results. Muons observed there, up to $\lg \left(N_{\mu}\right) \approx$ 5.0 , stem from hadronic interactions of energies below $200 \mathrm{GeV}$, modeled in simulations with FLUKA2002.4 code. At higher $\lg \left(N_{\mu}\right)$ - the lowest panel in Fig. 7 - contribution from higher interaction energies in this region increases. This suggests that low-energy interaction model FLUKA describes the data well. Muons produced above $\approx 3.5 \mathrm{~km}$ have parent mesons predominantly created at interaction energies larger than $200 \mathrm{GeV}$, which are modeled in simulations by the QGSJetII code. We observe here that the high-energy model has problems in describing the data.

The comparison reveals more muons at high production heights in the simulations (the distributions are shifted to the right). This excess of muons in the simulations at high altitudes with respect to the data may indicate that muons produced higher up have too high an energy and do not decay, surviving to the observation level, what is not observed in the measurements. The same effect will occur when the simulated mesons have too small an energy in the region of the first or second interaction, and would decay earlier than in reality. Shifting the maximum of shower development in the models deeper in the atmosphere will act in the direction of reducing the observed discrepancy with the data.

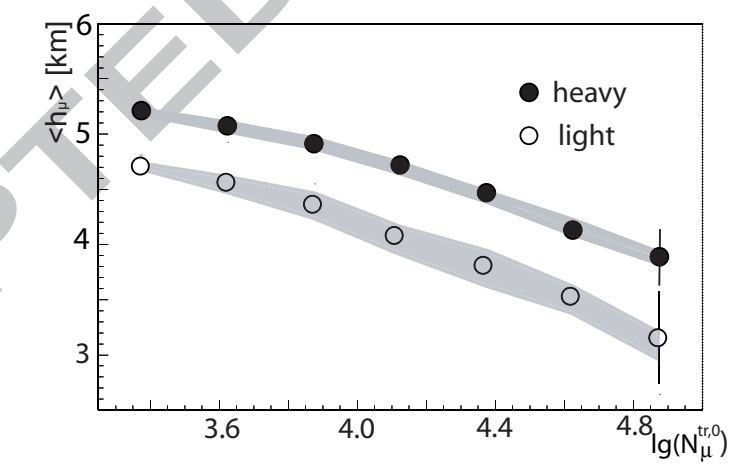

Figure 8: Experimental values of the mean muon production height along the shower axis $<h_{\mu}>$ vs. $\lg \left(N_{\mu}^{t r, 0}\right)$ for light and heavy primary mass enriched showers and a shower core distance range $40-80 \mathrm{~m}$, and shower angles $\theta<18^{\circ}$. The bands bracketing the data points represent variations for the $\lg \left(N_{\mu}^{t r, 0}\right) / \lg \left(N_{e}^{0}\right)$ ratio from 0.73 to 0.75 .

Simulation results shown in Fig. 7 are similar to the ones obtained with the older QGSJet01 model, which predicted the mean $h_{\mu}$ values shifted 
slightly up by $\approx 150 \mathrm{~m}$.

In Fig. 8 experimental values of the mean muon production height $\left\langle h_{\mu}\right\rangle$ as a function of $\lg \left(N_{\mu}^{t r, 0}\right)$ are shown. A good separation between light and heavy enriched primary CR particles is seen. The dependence on $\lg \left(N_{\mu}^{t r, 0}\right)$ suggests that production heights corrected for an appropriate elongation rate will exhibit a clear remaining dependence on the CR particle mass. Mean muon production heights for light and heavy mass enriched showers exhibit a height difference of about $12 \%$, which is $3-4$ times the systematic error quoted in Table 2.

Shaded bands show how $\left\langle h_{\mu}>\right.$ changes when the boundary between 'heavy' and 'light' is moved by \pm 0.01 in the $\lg \left(N_{\mu}^{t r, 0}\right) / \lg \left(N_{e}^{0}\right)$ ratio, which is $10-20 \%$ of the peak position difference in the distributions of this ratio for proton and iron primary induced showers [31]. One observes that the light sample is more sensitive to such a change giving a broader band, what can be explained by larger fluctuations in the shower development than in case of showers initiated by heavy CR primaries.

\section{Mean Muon Production Depth vs. Electron and Muon Shower Sizes}

In the previous section (Figs. 6, 7 and 8) the muon production height was shown to depend on the mass of the CR primary and its energy. In the following we will study this mass dependence of the muon production height in more detail. For this purpose we will use production height values referenced to the top of the atmosphere, called muon production depth, denoted as $H_{\mu}$, and expressed in units of atmospheric depth $\left(\mathrm{g} \cdot \mathrm{cm}^{-2}\right)$ using the USStandard atmosphere [38] in data - corrected for temperature and pressure and simulations. Seasonal differences between local atmospheric conditions near the KASCADE site and the US Standard are smaller than $\pm 2 \%$ (see Fig.2.1 in Ref. [38]) having little influence on our final results.

Usually, shower development is described by the evolution of its electromagnetic component, where $X_{\max }$ is understood as the atmospheric depth at which the number of electrons and photons of the air shower reaches its maximum. $X_{\max }$ is considered to be a primary mass sensitive parameter. Concerning muons which stem dominantly from $\pi^{ \pm}$decays, the corresponding production height at which most muons are created may also provide a mass sensitive observable. 
To reveal the primary mass sensitivity of the $H_{\mu}$ parameter one has to subtract the energy dependence expressed in terms of the elongation rate, taking into account the shower size observables.

Subtracting from the production depth $H_{\mu}$ for each track in a shower the energy dependent penetration depth we use the following relation for the mass dependent $H_{\mu}^{A}$

$$
H_{\mu}^{A}=H_{\mu}-D_{\mu}\left(\lg \left(N_{\mu}^{t r, 0}\right)-3.6\right)+D_{e}\left(\lg \left(N_{e}\right)-4.8\right)
$$

where the parameter offsets originate from the analysis thresholds.

For the following analysis the elongation rate components (the coefficients in equation (3)), similar to the energy dependence of $X_{\max }$ observed in EAS simulations, were assigned the following values: $D_{\mu}=70 \mathrm{~g} \cdot \mathrm{cm}^{-2}$ and $D_{e}=20 \mathrm{~g} \cdot \mathrm{cm}^{-2}$ per decade. These values have been varied iteratively by up to $\pm 20 \mathrm{~g} \cdot \mathrm{cm}^{-2}$ to obtain the slope of the middle $H_{\mu}^{A}$ ridge in the parameter $\lg \left(N_{e}^{0}\right)$ vs. $\lg \left(N_{\mu}\right)$ representation reproducing the $\lg \left(N_{\mu}\right) / \lg \left(N_{e}^{0}\right)$ ratio of heavy-light separation (Section 5). We assume that the elongation rates $D_{\mu}$ and $D_{e}$ are independent of the primary mass.

This two-component correction on $H_{\mu}$ is similar to the one for $\lg \left(E_{0}\right)$ in equation (1). There, contributions from $\lg \left(N_{e}\right)$ and $\lg \left(N_{\mu}\right)$, reflecting the complementary information on shower development, are also employed. The shower development leads also to different fluctuations in those shower parameters. It is known from earlier studies that the $\lg \left(N_{e}\right)$ parameter exhibits fluctuations to large values, in agreement with the simulations, while the $\lg \left(N_{\mu}^{t r}\right)$ parameter shows little fluctuations. On the contrary, the $H_{\mu}$ parameter (independent of shower angle) is fluctuating towards smaller values. Therefore, we may argue that in the corrections of $H_{\mu}$ for the elongation rate fluctuations will cancel to some extent and thus, the resulting mass dependent muon production depth $H_{\mu}^{A}$ represents a stable observable. In Fig. 9 muon production depth distributions are compared for three $N_{\mu}$ size bins before $\left(H_{\mu}\right)$ and after $\left(H_{\mu}^{A}\right)$ correction for the elongation rate, according to equation (3).

Using equation (3) the presentation shown in Fig. 10 has been obtained. Here $<H_{\mu}^{A}>$ represents the average mass dependent production depth $H_{\mu}^{A}$ per shower having at least one muon track in the MTD. Above the logarithmic muon number 5.5 an analysis from the Grande array data in a separate distance range is considered. As a result one can identify regions of different mass dependent mean muon production depths in the two-parameter space 


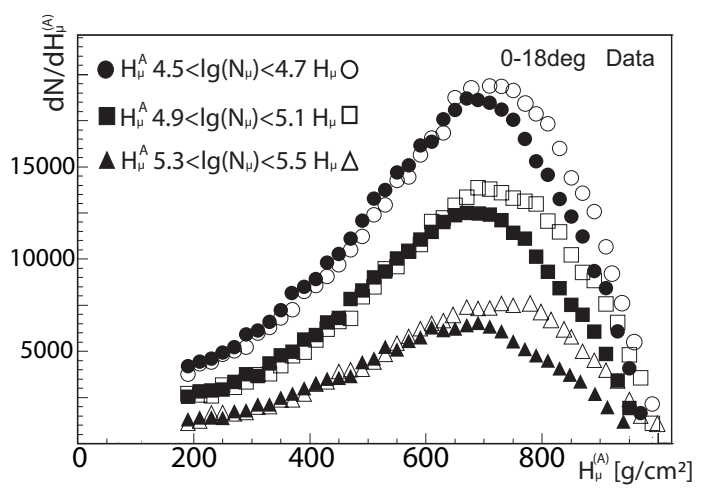

Figure 9: Muon production depth distributions are compared for three $\lg \left(N_{\mu}\right)$-size bins before (open symbols) $H_{\mu}$ and after (full symbols) $H_{\mu}^{A}$ correction for the elongation rate, according to the equation (3). The depth cut corresponds to $12 \mathrm{~km}$.

$\lg \left(N_{e}^{0}\right)$ vs. $\lg \left(N_{\mu}\right)$ which themselves vary over almost 3 orders of magnitude. These regions of distinct $\left\langle H_{\mu}^{A}\right\rangle$ are 'color' coded with a $40 \mathrm{~g} \cdot \mathrm{cm}^{-2}$ step size. Based on the assumption that the $\left\langle H_{\mu}^{A}\right\rangle$ parameter is not prone to large fluctuations, the spread of the regions of the same 'color' is a measure of the correlations in the two other fluctuating shower parameters $\lg \left(N_{e}^{0}\right)$ and $\lg \left(N_{\mu}\right)$.

The borders between different regions are marked for some cases with solid or dashed lines with a certain slope in the $\lg \left(N_{e}^{0}\right)$ vs. $\lg \left(N_{\mu}\right)$ plane. In the middle ridge the solid line has the previously employed slope for selecting light or heavy primary particles, being now equal to 0.83 instead of 0.74 , due to the transformation to $\lg \left(N_{\mu}\right)$. For regions away from the middle different slopes of the ridges may be observed, as in the case of the dashed line which marks the ridge of one of the heavier groups of primaries. Data boundaries are curved because of the limited statistics of showers and CR primary types, as shown in Fig. 22 in Ref. [26].

An integral number of muons for a nucleus $A$ induced shower is

$$
N_{\mu}^{A} \sim A\left(E_{0} / A\right)^{\beta}
$$

where $\beta \approx 0.85$ [53]. Taking into account that $X_{\max } \sim \lg \left(E_{0} / A\right)+$ const and assuming that $X_{\max , \mu}^{A}$ exhibits a similar $\lg \left(E_{0} / A\right)$ dependence as $X_{\max }$, using equation (4), we obtain: 


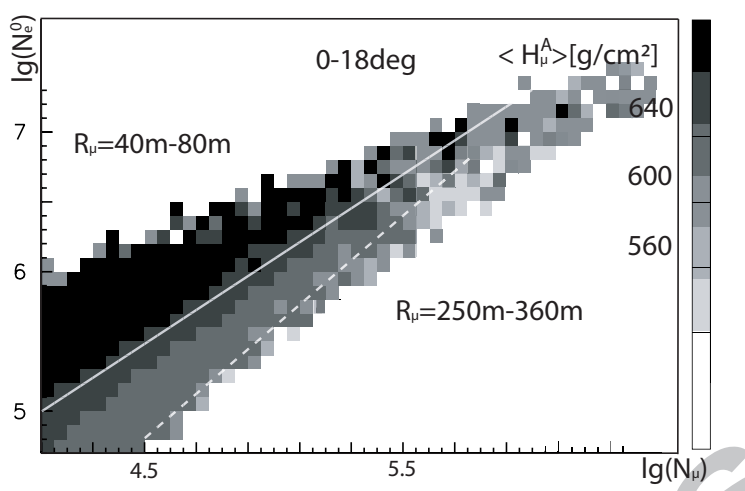

Figure 10: $\lg \left(N_{e}^{0}\right)$ vs. $\lg \left(N_{\mu}\right)$ matrix with effective mean muon production depth $\left\langle H_{\mu}^{A}\right\rangle$ along the $z$-axis. Borders between different regions are marked with solid or dashed lines (see text). Here, $\lg \left(N_{\mu}\right) \approx \lg \left(N_{\mu}^{t r, 0}\right)+0.5$ (see Section 3). Above $\lg \left(N_{\mu}\right)=5.5$ data from the Grande array in a distance range $250-360 \mathrm{~m}$ are considered and normalized to KASCADE data.

$$
X_{\text {max }, \mu}^{A} \sim 1 / \beta\left[\lg \left(N_{\mu}^{A}\right)-(1-\beta) \lg (A)\right] .
$$

Fig. 9 shows that in the $H_{\mu}^{A}$ range of our analysis $\left(>200 \mathrm{~g} \cdot \mathrm{cm}^{-2}\right), X_{\max , \mu}^{A}$ peak position) is larger than the $\left\langle H_{\mu}^{A}\right\rangle$ value, due to the tails in $H_{\mu}^{A}$ distributions towards the small values. However, this $X_{\max , \mu}^{A}$ in Fig. 9 is close to the largest $\left\langle H_{\mu}^{A}>\right.$ value in Fig. 10.

A scale for $<H_{\mu}^{A}>$ regions corresponding to 'iron' and 'proton' primaries can be estimated based on an analogy to the electromagnetic cascade, ignoring the difference between $X_{\max , \mu}^{A}$ and $\left\langle H_{\mu}^{A}\right\rangle$. For a $10^{16} \mathrm{eV}$ proton shower we obtain the number of hadronic cascade generations to be $n \approx 5.5$ from $X_{\max , \mu}^{p} \approx 660 \mathrm{~g} \cdot \mathrm{cm}^{-2}$ in Fig. 10, having in mind that $X_{\max , \mu}^{p}=n \cdot \lambda_{\pi-a i r} \approx 5.5$ $\cdot 120 \mathrm{~g} \cdot \mathrm{cm}^{-2} \approx 660 \mathrm{~g} \cdot \mathrm{cm}^{-2}$, and that a fraction of pions decay immediately into muons. For heavy CR particles we read from Fig. 10 a corresponding value $X_{\max , \mu}^{F e} \approx 540 \mathrm{~g} \cdot \mathrm{cm}^{-2} \approx 4.5 \cdot 120 \mathrm{~g} \cdot \mathrm{cm}^{-2}$, leading to 4.5 generations and providing the scale of equation (5). For proton primaries the normalization of equation (5) is achieved by setting $1 / \beta \approx 138 \mathrm{~g} \cdot \mathrm{cm}^{-2}$. The other extreme value for equation (5) for iron would lead to $X_{\max , \mu}^{F e} \approx X_{\max , \mu}^{p}-29.5$ $\mathrm{g} \cdot \mathrm{cm}^{-2} \cdot \ln (56) \approx 540 \mathrm{~g} \cdot \mathrm{cm}^{-2}$.

The slope of the lines in Fig. 10 can be also interpreted using the muon number vs. energy relation in equations (4) and (1). The exponent $\beta$ can 
be connected to the amount of inelasticity involved in the processes of $A$-air collisions [53]. A comparatively steeper slope observed in the dashed line may correspond to an increased inelasticity [53] with respect to the light CR primaries, which may be a subject of further studies.

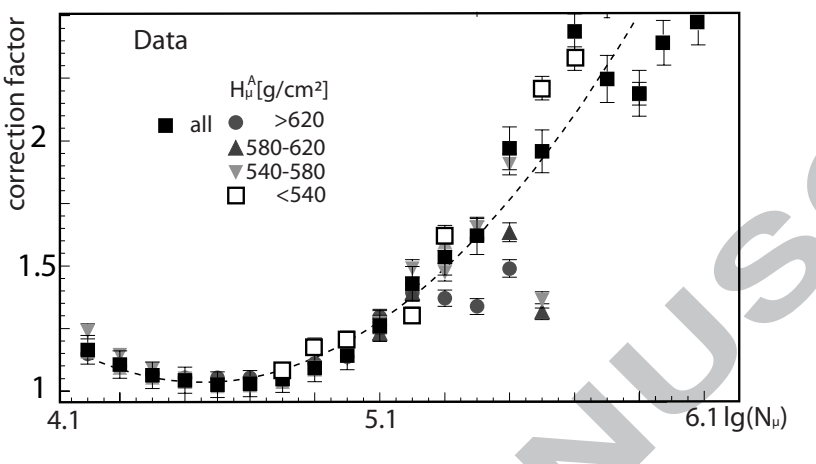

Figure 11: Flux correction factors due to the acceptance and the geometry differences between the array and the MTD (see text for details). The curve represents a polynomial fit.

To derive the CR flux spectra from the $\left\langle H_{\mu}^{A}>\right.$ information in Fig. 10 one has to introduce correction factors. They are obtained from the ratio of all registered showers which trigger KASCADE in the 40-80 m distance range to all showers with at least one track inside the MTD which survive the analysis cuts. Flux correction factors are shown in Fig 11. Correction values take into account the efficiency of the registration and reconstruction of muon tracks, as well as the geometry of the MTD. The size of the clusters on wires and strips in the MTD becomes large for high particle densities. Therefore, due to the software limit on these sizes (see Section 2) large showers are only accepted further away within the $40-80 \mathrm{~m}$ interval. These corrections are almost independent of the selection of $\left\langle H_{\mu}^{A}\right\rangle$ bins where the statistics is sufficient.

Sorting the $\lg \left(N_{e}^{0}\right)$ vs. $\lg \left(N_{\mu}^{t r}\right)$ events by regions of constant $\left\langle H_{\mu}^{A}\right\rangle$ in the matrix of Fig. 10, then multiplying their number by the flux correction factors, and applying the almost mass independent equation (1) for $\lg \left(E_{0}\right)$ the energy spectra of relative production depth abundances are obtained and shown in Fig. 12. So far, no explicit mass range assignment is given. The spectra are shown together with their systematic and statistical error combinations. The errors with wide boundary bars are statistical while the short 
boundary bars indicate the systematic variation of the $H_{\mu}^{A}$ regions between $\pm 20 \mathrm{~g} \cdot \mathrm{cm}^{-2}$ multiplied by the correction factors from Fig. 11. In Fig. 12 the Grande data, analyzed in the separate distance range (see Fig. 10), are normalized to KASCADE flux in two bins below and above $\lg \left(E_{0} / \mathrm{GeV}\right)=7.7$, i.e. in the overlapping region. The spectra reveal distinct features. While the 'low mass' ( $H_{\mu}^{A}$ large) spectra show a rapid drop with increasing shower energy, 'medium' and 'heavy mass' ( $H_{\mu}^{A}$ small) spectra seem to overtake at large primary energy. This feature is model independent because only the experimental data are used for obtaining mean muon production depth abundances.

In the present analysis - due to the detection threshold of the MTD - a small fraction of tracks $(\approx 15 \%)$ may be missing at small $\lg \left(N_{\mu}\right)$ (Fig. 11), leading to a bias in the light particle 'mass' interpretation. Unfortunately, our result cannot contribute to the discussion on the behavior of the total CR flux spectrum at higher energies, because the MTD detection area is too small and the amount of collected data limited. The required corrections from Fig. 11 would become prohibitively large. However, as indicated by the dashed line in Fig. 12, the total flux spectrum is in good agreement with the KASCADE spectrum obtained by an unfolding technique [26].

Table 3 provides a collection of the fluxes for different muon production depth $H_{\mu}^{A}$ windows showing results for every second bin in Fig. 12. The errors of the fluxes are dominated by systematic errors.

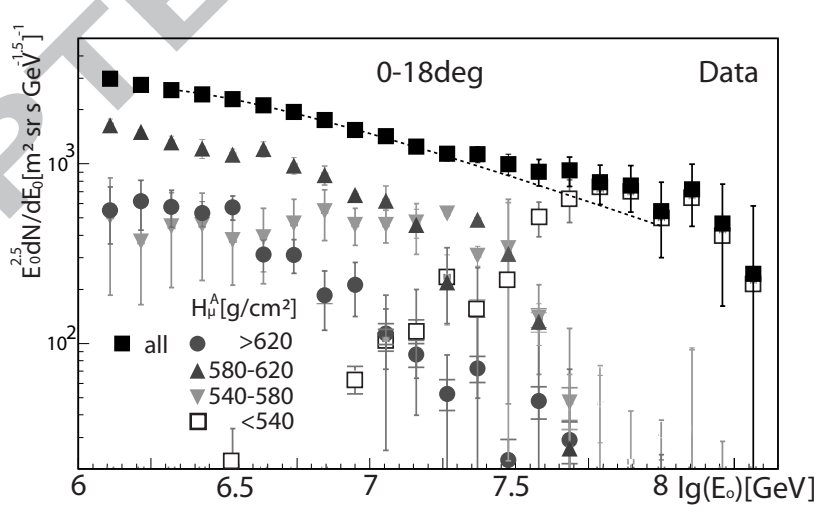

Figure 12: Energy spectra for primaries which produce muons at different effective muon production depth $H_{\mu}^{A}$; above $\lg \left(E_{0} / G e V\right)=7.7$ the Grande array data analysed in a separate distance range are considered. The dashed line reproduces the $\mathrm{CR}$ spectrum as measured by KASCADE - Ref. [26]. 


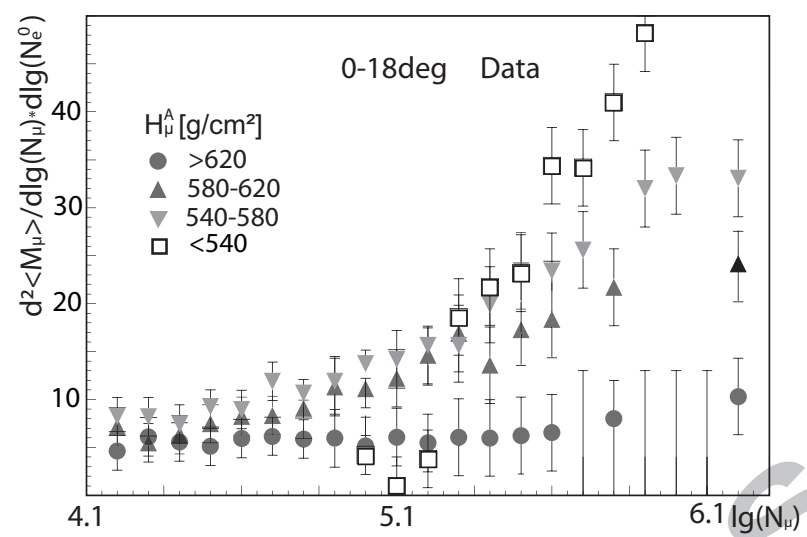

Figure 13: Differential muon multiplicity spectra in the MTD for different effective muon production depth $H_{\mu}^{A}$. Above $\lg \left(N_{\mu}\right)=5.5$ Grande multiplicities are normalized to KASCADE multiplicities in the overlapping region.

Each shower event is also characterized by a multiplicity of muon tracks. These multiplicities help improving the statistical accuracy of the $\left\langle H_{\mu}^{A}\right\rangle$ determination having no influence on the systematic uncertainties of the analysis described above. However, one finds that the mean muon multiplicity depends on the primary CR energy in a different way for different $<H_{\mu}^{A}>$ regions ( $\approx$ different primary mass groups), what is shown in Fig. 13. Double-differential mean muon multiplicities $d^{2}<M_{\mu}>/ d \lg \left(N_{\mu}\right) \times d \lg \left(N_{e}^{0}\right)$ detected by the MTD are selected for specific $\left\langle H_{\mu}^{A}\right\rangle$ regions (see Ref. [27] or Ref. [54] for comparable information). Data show variations due to the fluctuations in the $\lg \left(N_{e}^{0}\right)$ parameter. These multiplicities rise with $\lg \left(N_{\mu}\right)$ but with different slopes for different $\left\langle H_{\mu}^{A}\right\rangle$ regions. Therefore, the mean muon multiplicity rise with primary energy can be used for the primary CR mass estimation.

\section{Summary and Outlook}

The KASCADE-Grande experimental setup, where the electron and muon components of EAS are measured with high accuracy by arrays of scintillator detectors simultaneously with the precise measurement of muon directions in the tracking detector, allows us to approach the estimation of primary $\mathrm{CR}$ composition by means of investigation of the muon production heights in EAS. 
Table 3: Fluxes $\Phi$ and systematic errors $\Delta$ for different "mass" groups (in $\left.\left(\mathrm{m}^{2} \cdot \mathrm{sr} \cdot \mathrm{s} \cdot \mathrm{GeV}^{-1.5}\right)^{-1}\right)$.

\begin{tabular}{|c|c|c|c|c|c|c|c|c|}
\hline $\lg \left(E_{0}\right)[\mathrm{GeV}]$ & $\Phi_{620}$ & $\Delta_{620}$ & $\Phi_{600}$ & $\Delta_{600}$ & $\Phi_{560}$ & $\Delta_{560}$ & $\Phi_{540}$ & $\Delta_{540}$ \\
\hline 6.1 & 551.6 & 194.1 & 1641.4 & 130.5 & 510.4 & 324.6 & 0.0 & 0.0 \\
\hline 6.3 & 576.9 & 133.8 & 1316.4 & 109.3 & 447.5 & 243.1 & 3.4 & 0.0 \\
\hline 6.5 & 573.6 & 87.8 & 1124.7 & 83.3 & 375.6 & 164.9 & 22.2 & 11.4 \\
\hline 6.7 & 312.3 & 67.1 & 980.4 & 106.1 & 465.7 & 169.7 & 6.8 & 3.6 \\
\hline 6.9 & 211.7 & 70.8 & 669.7 & 34.7 & 458.4 & 105.5 & 63.5 & 0.0 \\
\hline 7.1 & 86.8 & 22.7 & 457.7 & 143.9 & 471.3 & 86.4 & 119.9 & 80.1 \\
\hline 7.3 & 72.8 & 78.6 & 489.4 & 10.4 & 308.5 & 39.4 & 157.2 & 107.6 \\
\hline 7.5 & 47.7 & 72.3 & 131.8 & 34.2 & 139.6 & 72.2 & 502.8 & 110.3 \\
\hline 7.7 & $<8.7$ & 5.0 & $<45.8$ & 31.8 & $<47.1$ & 19.1 & 717.6 & 194.8 \\
\hline 7.9 & $<7.3$ & 16.7 & $<48.9$ & 2.2 & $<30.9$ & 8.4 & 496.3 & 246.0 \\
\hline 8.1 & $<4.8$ & 14.6 & $<13.2$ & 6.9 & $<14.0$ & 14.5 & 424.1 & 305.0 \\
\hline
\end{tabular}

It has been shown that certain muon production depth $H_{\mu}^{A}$ regions create bands in the two parameter $\lg \left(N_{e}\right)$ vs. $\lg \left(N_{\mu}\right)$ space, which can be transformed into $\mathrm{CR}$ energy spectra in the range from $\approx 10^{15} \mathrm{eV}$ to $\approx 10^{17} \mathrm{eV}$ (Fig. 12). These spectra have features similar to the energy spectra of primary mass groups from Ref. [26]. One can state that the behavior of larger production depths $H_{\mu}$ describes the 'lighter' masses and the smaller production depths correspond to 'heavier' ones. A coarse mass scale may be provided by $X_{\max , \mu}^{A} \sim \ln (A)$.

Muon multiplicity distributions provide an extra parameter in determining the CR primary mass and future tests for high energy interaction models employed in EAS simulations.

Muons are direct messengers of hadronic interactions, and it is a common understanding that they are very well suited for testing the interaction models used in simulations and interpretations of the EAS data. The results obtained in this work are to some extent based on the QGSJet01 model (energy conversion formula (1)) and confirm the effect seen in the spectra in Ref. [26], namely, that the lightest masses (here the largest atmospheric depths) have a break in the spectrum at lower energies compared to the 
heavier (smaller depths) ones. However, the obtained relative abundances of various production depth ranges (Fig. 12) are model independent.

In addition to the investigation of EAS development using triangulation of muons the mean pseudorapidity of muons in $\operatorname{EAS}[47,52,55]$ is a very promising tool for tests of the interaction models. This parameter is closely related to the rapidity of pions - the most abundant products of high energy interactions - and it is highly sensitive to the longitudinal development of the shower in the atmosphere. Simulations show [47] that muons produced at a certain height $H_{\mu}$ carry to the observation level a certain mean pseudorapidity $<\eta>$, fairly independent from primary mass and energy. Therefore, $H_{\mu}$ provides an average measure of $\langle\eta\rangle$ and an excess of certain $H_{\mu}$ values can be related to the excess of certain pseudorapidity values in the production of mesons at given heights. KASCADE-Grande with its MTD offers the possibility of such investigation.

The discrepancy in the muon production height distributions between QGSJet simulations and measurements (Fig. 7) points to the necessity of further investigation of high energy interaction models.

Concerning the muon production height, shower zenith angle ranges larger than $18^{\circ}$ will be investigated in the near future. Also very inclined muons studied using the wall modules of the MTD are subject of ongoing investigations.

\section{Acknowledgements}

The authors would like to thank the members of the engineering and technical staff of the KASCADE-Grande collaboration, who contributed to the success of the experiment. Our special thanks go to all who over past years have contributed to the design and build-up of the MTD. The KASCADEGrande experiment is supported by the BMBF of Germany, the MIUR and INAF of Italy, the Polish Ministry of Science and Higher Education (this work by grant for the years 2009-2011), PPP-DAAD project for 2009-2010, and by the Romanian Authority for Scientific Research CNCSIS-UEFISCSU (grant PNII-IDEI no.461/2009, code 1442/2008 and project PN 093701 05).

\section{References}




\section{References}

[1] P.K.F. Grieder , Extensive Air Showers (Chapter 14), Springer Verlag GmbH, First edition, 2010.

[2] J. Gress et al., Nucl. Instr. and Meth. A 302 (1991) 368.

[3] O. Catalano et al, Nuovo Cim. C 15 (1992) 759.

[4] M. Feuerstack et al., Nucl. Instr. and Meth. A 315 (1992) 357.

[5] W. Rhode et al., Nucl. Instr. and Meth. A 378 (1996) 399.

[6] L. Horton et al., Nucl. Instr. and Meth. A 325 (1993) 326.

[7] S.K. Gupta et al., (GRAPES Coll.), Nucl. Phys. B (Proc. Suppl.) 196 (2009) 153.

[8] A. Petrukin et al., NEVOD-DECOR experiment, Symposium "Advances in Cosmic Ray Studies",Karlsruhe, March 2009, (http://wwwik.fzk.de/kascade-symposium/).

[9] S. Ahlen et al., MACRO-Coll., Nucl. Instr. and Meth. A 324 (1993) 337.

[10] T.K. Gaisser et al., IceCube Coll., Proc. 30 th ICRC, Merida, Mexico, arXiv:0711.0353, p.15-18.

[11] M. Ageron et al., ANTARES Coll., Astropart. Phys. 31 (2009) 277.

[12] D.G. Michael et al., MINOS Coll., Nucl. Instr. and Meth. A 596 (2008) 190.

[13] O. Gress et al., TUNKA Coll., Nucl. Phys. B (Proc.Suppl.) 75A (1999) 299.

[14] F. Arqueros et al., HEGRA Coll., Astron. Astrophys. 359 (2000) 682.

[15] S.P. Swordy and D.B. Kieda, Astropart. Phys. 13 (2000) 137.

[16] H. Falcke et al., LOPES Coll., Nature 435 (2005) 313; W.D. Apel et al., LOPES Coll., Astropart. Phys. 32 (2010) 294.

[17] R.M. Baltrusaitas et al., Nucl. Instr. and Meth. A 240 (1985) 410. 
[18] B. Keilhauer et al., AUGER Coll., Astropart. Phys. 25 (2006) 259.

[19] J. Milke et al., KASCADE Coll., Proc. $29^{\text {th }}$ ICRC 2005, Pune, India, Vol. 6, 125 .

[20] T. Antoni et al., KASCADE Coll., J. Phys. G: Nucl. Part. Phys. 34 (2007) 2581.

[21] L. Linsley, J .Phys. G: Nucl. Part. Phys. 12 (1986) 51; L. Linsley, Nuovo Cim. C 15 (1992) 743.

[22] M. Ambrosio et al, Nucl. Instr. and Meth. A 344 (1994) 350.

[23] T.V. Danilova et al., J. Phys. G: Nucl. Part. Phys. 20 (1994) 961.

[24] I.M. Brancus et al., Astropart. Phys. 7 (1997) 343.

[25] G. Navarra et al., KASCADE-Grande Coll., Nucl. Instr. and Meth. A 518 (2004) 207.

[26] T. Antoni et al., KASCADE Coll., Astropart. Phys. 24 (2005) 1.

[27] P. Doll et al., Nucl. Instr. and Meth. A 488 (2002) 517;

[28] T. Antoni et al., KASCADE Coll., Nucl. Instr. Meth. A 513 (2003) 490.

[29] J. Zabierowski and P. Doll., Nucl. Instr. and Meth. A 484 (2002) 528.

[30] C. Büttner et al., KASCADE Coll., Proc. 28 ${ }^{\text {th }}$ ICRC 2003, Tsukuba, Japan, Universal Academy Press Inc., HE 1.1, 33; C. Büttner, PhD Thesis, Report FZKA 6948, Forschungszentrum Karlsruhe (2004).

[31] R. Obenland, PhD Thesis, Report FZKA 7116, Forschungszentrum Karlsruhe (2005).

[32] R. Obenland et al., KASCADE Coll., Proc. 27 ${ }^{\text {th }}$ ICRC 2001, Hamburg, Germany, Vol. 2, 814.

[33] T. Antoni et al., KASCADE Coll., Astropart. Phys. 14 (2001) 245.

[34] A. Chiavassa et al., KASCADE-Grande Coll., Proc. $21^{\text {st }}$ ECRS, Kosice, Slovakia, 2008, Inst. of Exp. Phys. Slovac Academy of Sciences, (2009) 437. 
[35] W.D. Apel, et al. (KASCADE-Grande Coll.), Nucl. Instr. and Meth. A 620 (2010), 202.

[36] P. Doll et al., KASCADE-Grande Coll., Proc. 31 ${ }^{\text {st }}$ ICRC, 2009. Lodz, Poland, Report FZKA 7516, Forschungszentrum Karlsruhe (2009) 45.

[37] J.H. Weber et al., KASCADE Coll., Proc. 25 th ICRC 1997, Durban, South Africa, Vol. 6, 153.

[38] D. Heck et al., FZKA-Report 6019, Forschungszentrum Karlsruhe (1998).

[39] N.N. Kalmykov and S. Ostapchenko and A.I. Pavlov, Nucl. Phys. B (Proc.Suppl.) 52B (1997) 17; S. Ostapchenko, Phys. Rev. D 74, (2006) 014026.

[40] R. Glasstetter et al.,KASCADE Coll., Proc. 29 th ICRC 2005, Pune, India, Vol. 6, 101.

[41] A. Haungs et al., Proc. $31^{\text {st }}$ ICRC, 2009. Lodz, Poland, Report FZKA 7516, Forschungszentrum Karlsruhe, (2009) 1.

[42] K. Bernlöhr, Astropart.Phys. 5 (1996) 139.

[43] J. Zabierowski, K. Daumiller and P. Doll, Nucl. Phys. B (Proc. Suppl.) $122(2003) 275$.

[44] L. Pentchev, P. Doll and H.O. Klages, J.Phys.G: Nucl. Part. Phys. 25 (1999) 1235; L. Pentchev and P. Doll, J. Phys. G: Nucl. Part. Phys. 27 (2001) 1459.

[45] C. Büttner et al., KASCADE Coll., Nucl. Phys. B (Proc. Suppl) 122 (2003) 289.

[46] M. Ambrosio, C. Aramo, L. Colesanti, A.D. Erlykin, S.K. Machavariani, J. Phys. G: Nucl. Part. Phys. 23 (1997) 219.

[47] J. Zabierowski et al., KASCADE Coll., Proc. 31 ${ }^{\text {st }}$ ICRC, 2009. Lodz, Poland, Report FZKA 7516, Forschungszentrum Karlsruhe, (2009) 37.

[48] H. Fesefeldt, Report PITHA-85/02, RWTH Aachen, 1985. 
[49] T. Antoni et al., KASCADE Coll., Astropart. Phys. 19 (2003) 703.

[50] A. Fasso et al., CERN-2005-10, INFN/TC-05/11, SLAC-R-773, 2005; A. Fasso et al., arXiv:hep-ph/0306267.

[51] R. Brun and F. Carminati, GEANT Detector Description and Simulation Tool, CERN Program Library Long Writeup (1993).

[52] J. Zabierowski et al., KASCADE Coll., Nucl. Phys. B (Proc. Suppl.) 151 (2006) 291.

[53] J. Matthews, Astropart. Phys. 22 (2005) 387.

[54] M. Bertaina et al., EAS-TOP Coll., Proc. $29^{\text {th }}$ ICRC 2005, Pune, India, Vol. 6, 41.

[55] J. Zabierowski et al., KASCADE Coll., Proc. 29 ${ }^{\text {th }}$ ICRC 2005, Pune, India, Vol. 6, 357. 


\section{Figure captions for the article}

Fig. 1. Anode wire amplitude spectra for individual odd/even numbered wire cells.

Fig. 2. Illustration of the angular correlations in EAS and the definition of the radial $(\rho)$ and tangential $(\tau)$ angles. The differences between the true and the reconstructed $h_{\mu}$ values with the two reconstruction approaches - see text - are also shown.

Fig. 3. An example of radial angle distributions from Ref. [31] shows the behavior of this quantity in a wide range of $\lg \left(N_{\mu}^{t r}\right)$, i.e. of primary energy. The selection used comprises a radial distance range 40-120 m, shower zenith angles $\theta<30^{\circ}$ and $|\tau|<0.7^{\circ}$. Relative yields reflect the CR flux in different $\lg \left(N_{\mu}^{t r}\right)$ intervals. Lines connect the data points to guide the eyes.

Fig. 4. Mean muon production heights observed at different distances from the shower core in CORSIKA simulated vertical showers for proton and iron primaries and two fixed primary energy values [47].

Fig. 5. An example of muon production height distributions from Ref. [31] shows the behavior of this quantity in a wide range of $\lg \left(N_{\mu}^{t r}\right)$, i.e. primary energy. The selection used comprises a radial distance range of 40-120 m and shower zenith angles $\theta<30^{\circ}$. Radial and tangential angle cuts are the same as used in this article $\left(\rho=0-8^{\circ}\right.$ and $\left.|\tau|<0.7^{\circ}\right)$. Relative yields reflect the CR flux in different $\lg \left(N_{\mu}^{t r}\right)$ intervals. Lines connect the data points to guide the eyes.

Fig. 6. Radial angle distributions (left panel) and muon production height distributions $h_{\mu}$ along the shower axis (right panel) for light and heavy CR primary mass enriched showers and for different $\lg \left(N_{\mu}^{t r, 0}\right)$ intervals. Shower core distances range is $40-80 \mathrm{~m}$, and shower angles $\theta<18^{\circ}$. Lines connect the data points to guide the eyes.

Fig. 7. Muon production height distributions along the shower axis $h_{\mu}$ for light (open circles) and heavy (full circles) enriched shower samples and a shower core distance range $40-80 \mathrm{~m}$, and shower zenith angles $\theta<18^{\circ}$ compared to CORSIKA simulations employing QGSjetII +FLUKA models for proton $(\mathrm{H})$ and iron $(\mathrm{Fe})$ primaries. Here $\lg \left(N_{\mu}\right)=\lg \left(N_{\mu}^{t r, 0}\right)+0.5$ (see Section 
3). Lines connect the data points to guide the eyes.

Fig. 8. Experimental values of the mean muon production height along the shower axis $<h_{\mu}>$ vs. $\lg \left(N_{\mu}^{t r, 0}\right)$ for light and heavy primary mass enriched showers and a shower core distance range $40-80 \mathrm{~m}$, and shower angles $\theta<18^{\circ}$. The bands bracketing the data points represent variations for the $\lg \left(N_{\mu}^{t r, 0}\right) / \lg \left(N_{e}^{0}\right)$ ratio from 0.73 to 0.75 .

Fig. 9. Muon production depth distributions are compared for three $\lg \left(N_{\mu}\right)$-size bins before (open symbols) $H_{\mu}$ and after (full symbols) $H_{\mu}^{A}$ correction for the elongation rate, according to the equation (3). The depth cut corresponds to $12 \mathrm{~km}$.

Fig. 10. $\lg \left(N_{e}^{0}\right)$ vs. $\lg \left(N_{\mu}\right)$ matrix with effective mean muon production depth $<H_{\mu}^{A}>$ along the $z$-axis. Borders between different regions are marked with solid or dashed lines (see text). Here, $\lg \left(N_{\mu}\right) \approx \lg \left(N_{\mu}^{t r, 0}\right)+0.5$ (see Section 3). Above $\lg \left(N_{\mu}\right)=5.5$ data from the Grande array in a distance range $250-360 \mathrm{~m}$ are considered and normalized to KASCADE data.

Fig. 11. Flux correction factors due to the acceptance and the geometry differences between the array and the MTD (see text for details). The curve represents a polynomial fit.

Fig. 12. Energy spectra for primaries which produce muons at different effective muon production depth $H_{\mu}^{A}$; above $\lg \left(E_{0}\right)=7.7$ the Grande array data analysed in a separate distance range are considered. The dashed line reproduces the CR spectrum as measured by KASCADE - Ref. [26].

Fig. 13.Differential muon multiplicity spectra in the MTD for different effective muon production depth $H_{\mu}^{A}$. Above $\lg \left(N_{\mu}\right)=5.5$ Grande multiplicities are normalized to KASCADE multiplicities in the overlapping region. 